\title{
Photoluminescence and modulation spectroscopy study of the effects of growth interruptions on the interfaces of GaAsSb/GaAs multiple quantum wells
}

\author{
H. P. Hsu ${ }^{1}$, P. Sitarek ${ }^{* *}$, , Y. S. Huang ${ }^{*,}$, P. W. Liu ${ }^{2}$ J. M. Lin ${ }^{2}$, H. H. Lin ${ }^{2}$, \\ and K. K. Tiong ${ }^{3}$ \\ ${ }^{1}$ Department of Electronic Engineering, National Taiwan University of Science and Technology,
Taipei 106, Taiwan
${ }^{2}$ Department of Electrical Engineering, National Taiwan University, Taipei 106, Taiwan
${ }^{3}$ Department of Electrical Engineering, National Taiwan Ocean University, Keelung 202, Taiwan
}

Received 13 September 2006, revised 7 December 2006, accepted 11 December 2006

Published online 6 February 2007

PACS 78.20.Ci, 78.55.Cr, 78.67.De

Temperature dependent photoluminescence (PL), room temperature phototransmittance (PT), contactless electroreflectance (CER) and wavelength modulated surface photovoltage spectroscopy (WMSPS) measurements are utilized to study the effects of growth interruption times, combined with Sb exposure of $\mathrm{GaAsSb} / \mathrm{GaAs}$ multiple quantum wells (MQWs) structures. The PL spectra show peak location redshifted, luminescence intensity increased and full width at half maximum narrowed with increasing interruption time. The features originated from different regions of the samples including interband transitions of MQWs, interfaces and GaAs are observed and identified through a detailed comparison of the obtained spectra and theoretical calculation. The anomalous temperature dependence of PL spectra has been attributed to the carrier localization effect resulted from the presence of Sb clusters and/or fluctuations in $\mathrm{Sb}$ composition at the QW interfaces. An additional feature has also been observed below the GaAs region in $\mathrm{Sb}$ exposure treated samples and the probable origin of this feature has been discussed.

(C) 2007 WILEY-VCH Verlag GmbH \& Co. KGaA, Weinheim

\section{Introduction}

$\mathrm{GaAsSb} / \mathrm{GaAs}$ quantum well (QW) structure system is of significant interest due to its potential applications in novel heterostructure devices. Particularly, its band alignment provides an excellent opportunity to improve the performance of both heterojunction bipolar transistors [1] and optoelectronic devices [24]. Recently, devices using this system have been successfully developed, such as the fabrication of sources and detectors in long wavelength for fiber-optics communications [5], photodiodes [6], photocathodes [7], light-emitting diodes [8], and vertical cavity surface emitting lasers [9]. Besides its technological potential, it also serves as a model system for investigating the atomic ordering and compositional modulation expected in III- $-\mathrm{V}_{\mathrm{A}}-\mathrm{V}_{\mathrm{B}}$ alloys [10]. In spite of its importance, it is noticed that there are some difficulties in growing high-quality samples [11,12]. The specific difficulties in molecular beam epitaxial growth (MBE) of this structure arise as less than unity sticking coefficients of group V species complicates the precise composition control of GaAsSb layer and excess amount of $\mathrm{As}$ or $\mathrm{Sb}$ are still present on the surface even after As or $\mathrm{Sb}$ fluxes are closed. This makes control of abrupt interfaces

* Corresponding author: e-mail: ysh@mail.ntust.edu.tw, Phone: + 8862 27376385, Fax: + 886227376424

** Permament address: Institute of Physics, Wrocław University of Technology, Wyb. St. Wyspiańskiego 27, 50-370 Wrocław, Poland 
difficult due to incorporation of an uncommon group- $\mathrm{V}$ element, $\mathrm{Sb}$. The atomic configuration of these heterostructures at the interfaces can influence the optical and electronic properties of these devices due to changes in the QW profile.

In the present study, the effects of the growth interruptions at interfaces combined with Sb exposure of $\mathrm{GaAsSb} / \mathrm{GaAs}$ multiple quantum well (MQWs) structures have been characterized by temperature dependent photoluminescence (PL), room temperature phototransmittance (PT), contactless electroreflectance (CER), and wavelength modulated surface photovoltage spectroscopy (WMSPS). The PL spectra show red-shifted peaks with increasing intensity and narrowed full width at half maximum (FWHM) with increased of interruption time. The anomalous temperature dependence of PL spectra has been attributed to the carrier localization effect resulting from the presence of $\mathrm{Sb}$ clusters or fluctuations in $\mathrm{Sb}$ composition at the QW interfaces. An additional feature has also been observed below the GaAs region in $\mathrm{Sb}$ exposure treated samples. The possible origin of the red-shifts and additional feature are discussed.

\section{Experimental}

The three GaAsSb/GaAs multiple quantum well (MQWs) samples employed in this study were grown on semi-insulating GaAs(100) substrates using a VG V-80MKII molecular beam epitaxy (MBE). The $\mathrm{Sb}_{1}$ source was supplied using an EPI model 175 standard crackers $\mathrm{K}$ cell, and the $\mathrm{As}_{2}$ source was supplied from a Riber VAC 500 As valve cracker. The cracker zone temperature was $1050{ }^{\circ} \mathrm{C}$, while the bulk zone temperature was about $430{ }^{\circ} \mathrm{C}$. The structure of MQWs contains a $500 \mathrm{~nm}$ GaAs buffer layer, five periods of GaAsSb $6 \mathrm{~nm} / \mathrm{GaAs} 34 \mathrm{~nm}$. The growth of GaAsSb/GaAs MQWs proceeded as follows. During the growth, the cell shutter of Ga was kept open as long as necessary for the growth of the intended thickness of individual layers, while $\mathrm{Sb}$ termination was provided at the well/barrier interfaces. The interface exposure times were 10 and $30 \mathrm{~s}$ for samples II and III, respectively. In order to study the influence of $\mathrm{Sb}$ treatments, a specimen (sample I) was also grown without any interruption. The samples were terminated with a $100 \mathrm{~nm}$-thick GaAs cap layer and all the layers were unintentionally doped. The growth conditions of these three samples were identical except for the Sb exposure times. The antimony composition in the GaAsSb layers was determined to be $0.18,0.20$ and 0.21 for samples I, II and III, respectively, by double crystal X-ray diffraction (DXRD) measurements.

PL excitation was produced by a $20 \mathrm{~mW}, 532 \mathrm{~nm}$ Nd-YAG green laser. Luminescence was analyzed with a $0.35 \mathrm{~m}$ grating spectrometer and detected by an InGaAs detector using conventional lock-in detection. A CTI model 22 closed-cycle cryogenic refrigeration system equipped with a model 32B digital temperature controller was used for temperature dependent measurements. The measurements were made between 10 and $300 \mathrm{~K}$ with a temperature stability of $0.5 \mathrm{~K}$ or better. PT measurement was achieved using an internally modulated $5 \mathrm{~mW}$ laser diode $(670 \mathrm{~nm})$ as the modulating source. The laser intensity was reduced to about $1-10 \%$ of its initial value by using a neutral density filter. The probe beam was incident through the front surface of the sample. A $150 \mathrm{~W}$ tungsten-halogen lamp filtered by a PTI $0.25 \mathrm{~m}$ monochromator provided the monochromatic light. The transmitted light was detected by an InGaAs photodetector placed behind the sample, and the signal was recorded by a lock-in amplifier. The dc output of the photodetector was maintained constant by a servo mechanism of variable neutral density filter. For CER, an ac modulating voltage $(\sim 1 \mathrm{kV}$ at $200 \mathrm{~Hz})$ was applied between a front wire grid electrode and a second electrode consisting of a metal plate [13]. These two electrodes were separated by an insulating spacing which provided a very thin layer $(0.1 \mathrm{~mm})$ of air between the front surface of the sample and the front electrode. The configuration is nondestructive as there is no direct contact with the front surface of the sample. The reflected light was detected by a silicon photodiode detector and an InGaAs photodetector for the visible and near infrared region, respectively. For WMSPS, the derivative-like surface photovoltage was measured between the sample and a reference metal grid electrode in a capacitive manner as a function of the photon energy of the probe beam with a wavelength-modulation technique. The illumination system consisted of a $150 \mathrm{~W}$ quartz-halogen lamp and a grating monochromator equipped with a vibrating exit slit operated by a power amplifier, employing a 2 inch loudspeaker as the transducer. A beam splitter was placed in the path of the incident light. The intensity of this radiation 
was monitored by a pyroelectric detector and was kept a constant level of $\sim 10^{-5} \mathrm{~W} / \mathrm{cm}^{2}$ by a stepping motor connected to a variable neutral density filter, which was also placed in the path of the incident beam. The illumination intensity and the amplitude of wavelength modulation were experimentally selected at levels not affecting the measured spectra; typically $\Delta \lambda / \lambda$ was on the order of $10^{-3}$. Precautions have been taken to eliminate the spurious signals from light-intensity modulation. The normalized wavelength-modulated derivative-like photovoltage spectrum on the metal grid was measured with a copper plate as the ground electrode, using a buffer circuit and a lock-in amplifier.

\section{Results and discussion}

Figure 1 shows the PL spectra of GaAsSb/GaAs MQWs with Sb interface treatment at room temperature. As indicated in Fig. 1, compared to the sample grown without Sb treatment (sample I), samples II and III have longer emission wavelength and stronger PL intensity. The increase of the Sb content in MQWs may cause the ground transition energy red-shift in GaAsSb/GaAs MQWs and the results of the stronger luminescence of PL feature might indicate a better quality of the MQWs structures with $\mathrm{Sb}$ treatment.

Displayed in Fig. 2 is the temperature dependent peak position of PL spectra of samples I, II, and III in the temperature range from 10 to $300 \mathrm{~K}$. The PL peak position shifts to lower energy between 10 and $25 \mathrm{~K}$, and then increases between 25 and $50 \mathrm{~K}$. At the temperature above $75 \mathrm{~K}$, the peak position shifts again to lower energy. This S-shape phenomenon of temperature dependent PL at low temperature region was also observed on dilute nitride compounds $[14,15]$. This anomalous temperature dependence of PL peak position can be explained in terms of the competition between the transfer dynamics of the localized carrier/excitions and thermalization effect $[16,17]$. At low temperature the PL signal originates from the localized excitons induced by the nonuniform distribution of Sb clusters. With increase of temperature, some of the localized excitons may be thermally activated, and thus are mobile to some extent. Consequently, the peak energy of the thermal equilibrium distribution of the localized excitons will increase with increasing temperature and approach that of the delocalized excitons. This process can explain the blueshift of PL peak positions in the low temperature range. At still higher temperature, the PL signals mainly originate from the delocalized states and the peak energy red-shifts with thermal shrinkage of the energy gap of the well and barrier materials.

Figure 3 plots the FWHM of samples I, II, and III. The FWHMs of samples increase between 10 and $45 \mathrm{~K}$, and then slightly decrease between 45 and $100 \mathrm{~K}$. After $100 \mathrm{~K}$ the FWHMs increase with temperature. The anomalous behavior of FWHM can be explained in terms of competition between the localized and delocalized states $[18,19]$. The large FWHM of PL spectra results from the coexistence and competition between the localized and delocalized states. The localized states dominate on the low temperature domain $(<100 \mathrm{~K})$ while the delocalized states dominate over the higher temperature domain.

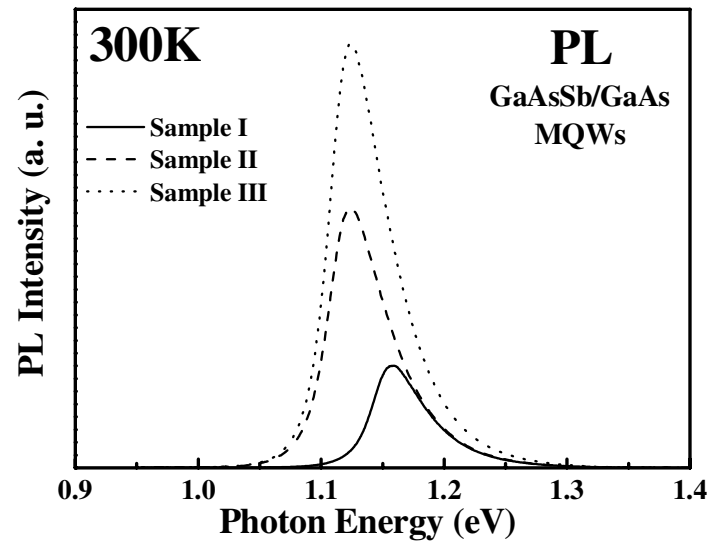

Fig. 1 PL spectra of $\mathrm{GaAs}_{1-r} \mathrm{Sb}_{2} / \mathrm{GaAs}$ samples at room temperature. 


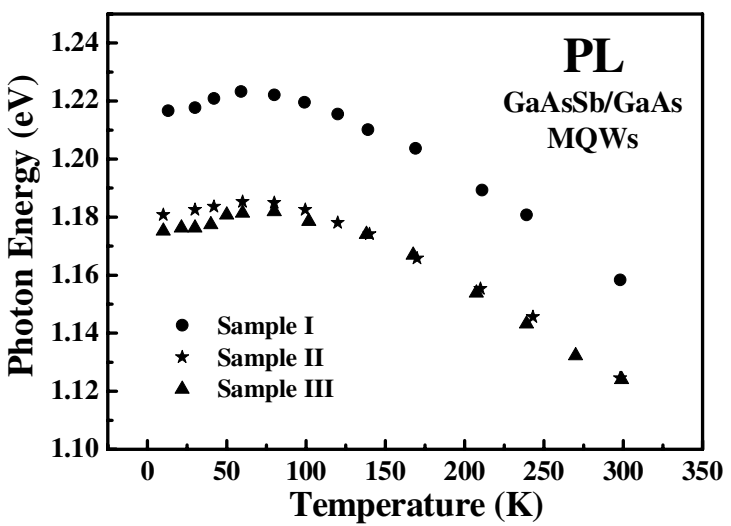

Fig. 2 PL peak energies versus temperature of $\mathrm{GaAs}_{1-x} \mathrm{Sb}_{x^{\prime}} / \mathrm{GaAs} \mathrm{MQWs}$ samples.

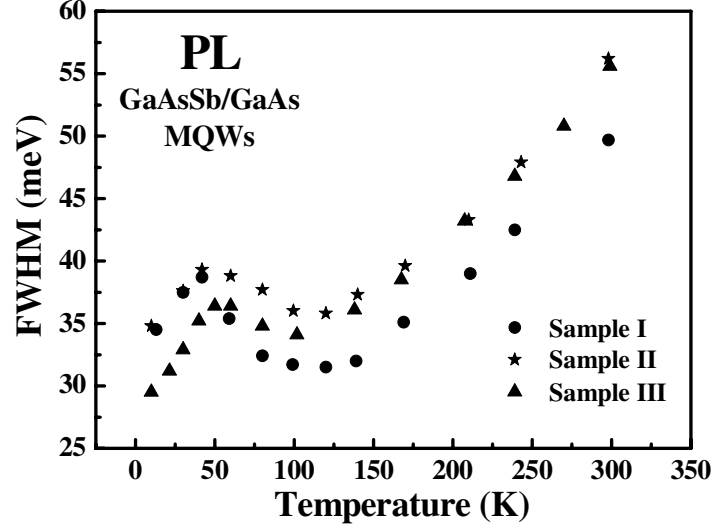

Fig. 3 PL FWHM as a function of temperature on $\mathrm{GaAs}_{1-x} \mathrm{Sb}_{x} / \mathrm{GaAs} \mathrm{MQWs}$ samples.

Figure 4 shows the schematic diagram of the band edges vs. growth direction for a compressively strained $\mathrm{GaAsSb} / \mathrm{GaAs} \mathrm{MQWs}$ strucutre. In contrast to the type-I semiconductor system, spatially separated electrons and holes are easily realized in such a type-II system, in which electrons are confined in the GaAs layer and holes are localized in the GaAsSb layer [11]. The indirect transitions due to the spatially separated electrons and holes are restricted in a very narrow region near the interfaces.

Shown by the dashed lines in Fig. 5 are the PT spectra in the region of 1.05 to $1.35 \mathrm{eV}$ at room temperature from the three GaAsSb/GaAs MQWs samples. The solid lines in Fig. 5 are least squares fits to the first-derivative Lorentzian lineshape (FDLL) function of the form [20, 21]

$$
\frac{\Delta T}{T}=\operatorname{Re} \sum_{j=1} A_{j} \mathrm{e}^{i \Phi_{j}}\left(E-E_{j}+i \Gamma_{j}\right)^{-n},
$$

where $A_{j}$ and $\Phi_{j}$ are the amplitude and phase of the line shape, $E_{j}$ and $\Gamma_{j}$ are the energy and broadening parameters of the excitonic transitions, and the value of $n$ depends on the origin of the transitions. For the first derivative functional form, $n=2$ is appropriate for the bound states such as excitons [21, 22]. The obtained interband energies are denoted by the letters A and B which correspond, respectively, to $11 \mathrm{H}$

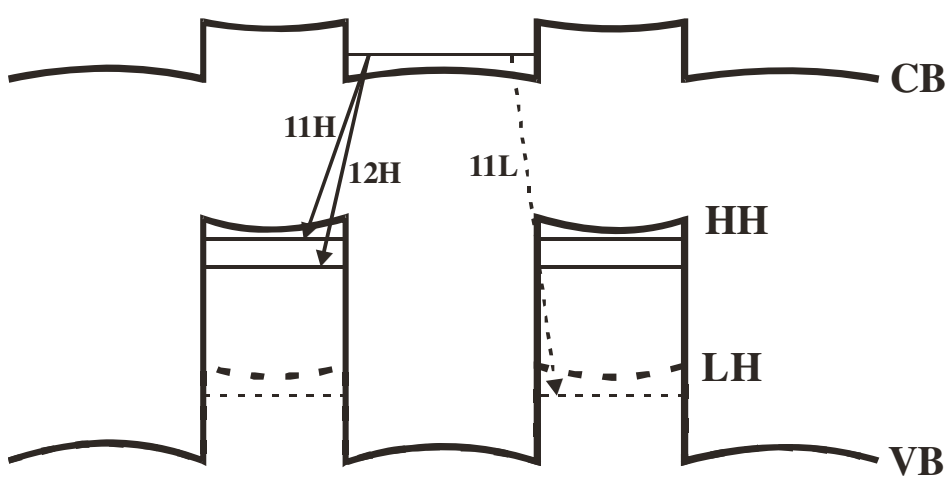

\section{GaAsSb GaAs GaAsSb}

Fig. 4 Schematic diagram of the band edges vs. growth direction for a compressively strained $\mathrm{GaAsSb} / \mathrm{GaAs}$ MQWs structure, where the QW with the relevant HH (solid lines) and LH (dashed line) transitions are indicated. 


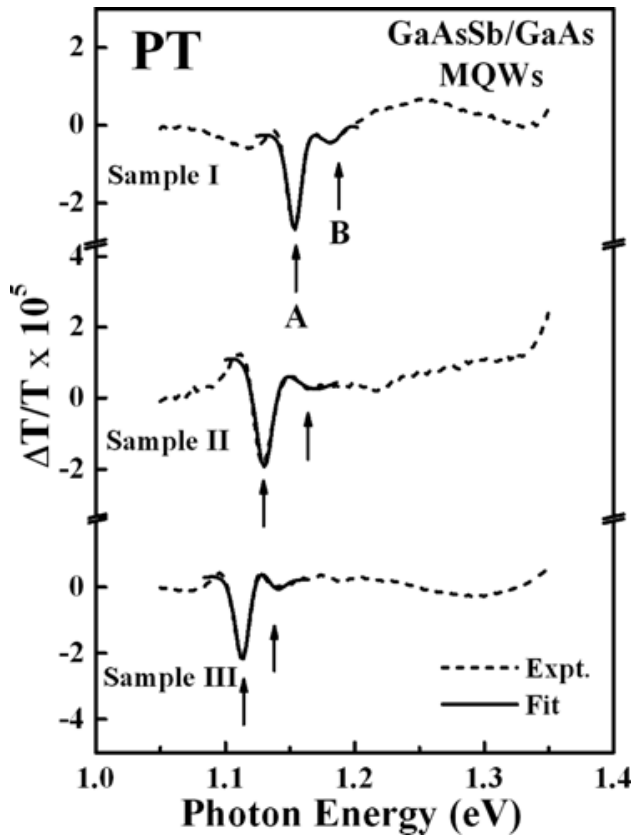

Fig. 5 PT spectra (dashed lines) from three $\mathrm{GaAs}_{1-x} \mathrm{Sb}_{x} / \mathrm{GaAs}$ samples taken at $300 \mathrm{~K}$. The solid lines are the least-squares fits to the FDLL line shape function. The obtained energy values are denoted by arrows.

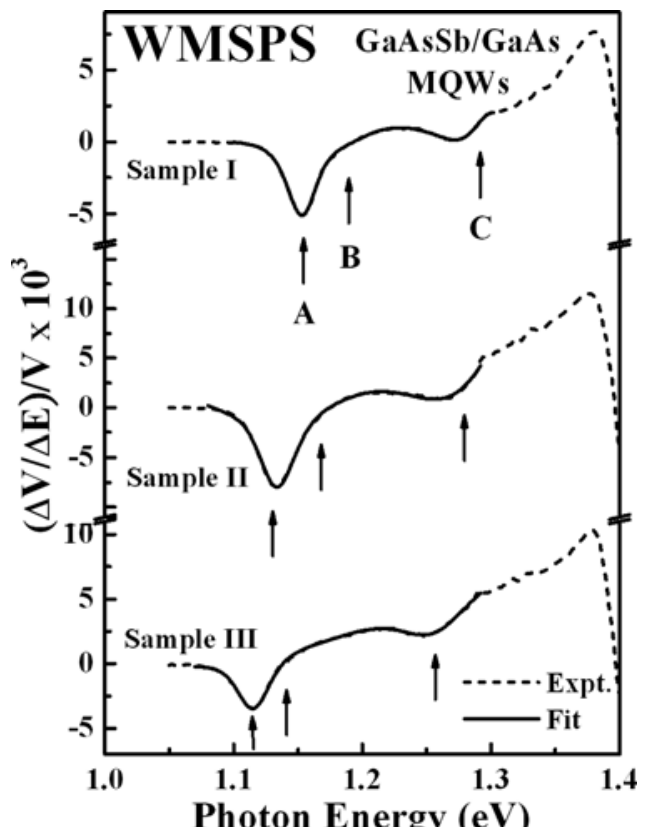

Fig. 6 WMSPS spectra (dashed lines) from three $\mathrm{GaAs}_{1-x} \mathrm{Sb}_{x} / \mathrm{GaAs}$ samples taken at $300 \mathrm{~K}$. The solid lines are the least-squares fits to the FDLL line shape function. The obtained energy values are denoted by arrows.

(first electron to first heavy-hole) and $12 \mathrm{H}$ (first electron to second heavy-hole), and their respective locations are marked by arrows below the features and listed in Table 1. Obtained fundamental transition $(11 \mathrm{H})$ energy values agree well with PL data presented in Fig. 1. It is clear that these two quantum-well related features shift toward lower energies with the increase of the interruption time (Sb exposure time). The observed energy red-shifts for Sb-treated samples can be understood to be due to increased bandalignment sensitivity toward the heterointerface state of these structures. Interface grading modifies the confining potential and involves complicated strain relief relaxation in the well. It has been demonstrated that heterostructures for both group III and group V species differ in sublayers [22, 23], local strain is present at the interfaces. This may lead to modifications of the band lineup due to the contribution of interface dipoles formed by the bond length variations along heterointerfaces such as $\mathrm{Ga}-\mathrm{Sb}$ or $\mathrm{Ga}-\mathrm{As}$. The formation of the intermixed layers will further modify the actual band alignment through electrons and holes confinements at the interfaces. Thus, the width and composition of the intermixed layers at the interfaces will influence the position and the linewidth of the interband transition features of the PT spectra. Compositional variations along the heterointerfaces as well as variations in size of the intermixed areas may be responsible for the linewidth increase observed in the samples with the Sb-exposure treatment.

Figure 6 shows the WMSPS spectra of the three samples at room temperature. The solid lines are the least-squares fits to the FDLL function [20, 21]. Note that the spectra on the lower energy sides are asymmetric. In order to have a reasonable fit of these regions two interband transitions are needed. The obtained energies are designated by arrows. The fitted values are summarized in Table 1 . The transition energies red shifted with the increase of Sb exposure time. In contrast to the PT spectra, the WMSPS spectra contain an additional broad feature, denoted by $\mathrm{C}$, located at about $140 \pm 10 \mathrm{meV}$ above the $11 \mathrm{H}$ transitions. In general, we would expect PT to be the more sensitive of these two techniques. However it is not the case in the present study. One possible reason might be due to the different modulation mecha- 
Table 1 Experimental and theoretical calculated values of the various interband transition energies of $\mathrm{GaAs}_{1-\mathrm{x}} \mathrm{Sb}_{x} / \mathrm{GaAs} \mathrm{MQWs}$ at $300 \mathrm{~K}$.

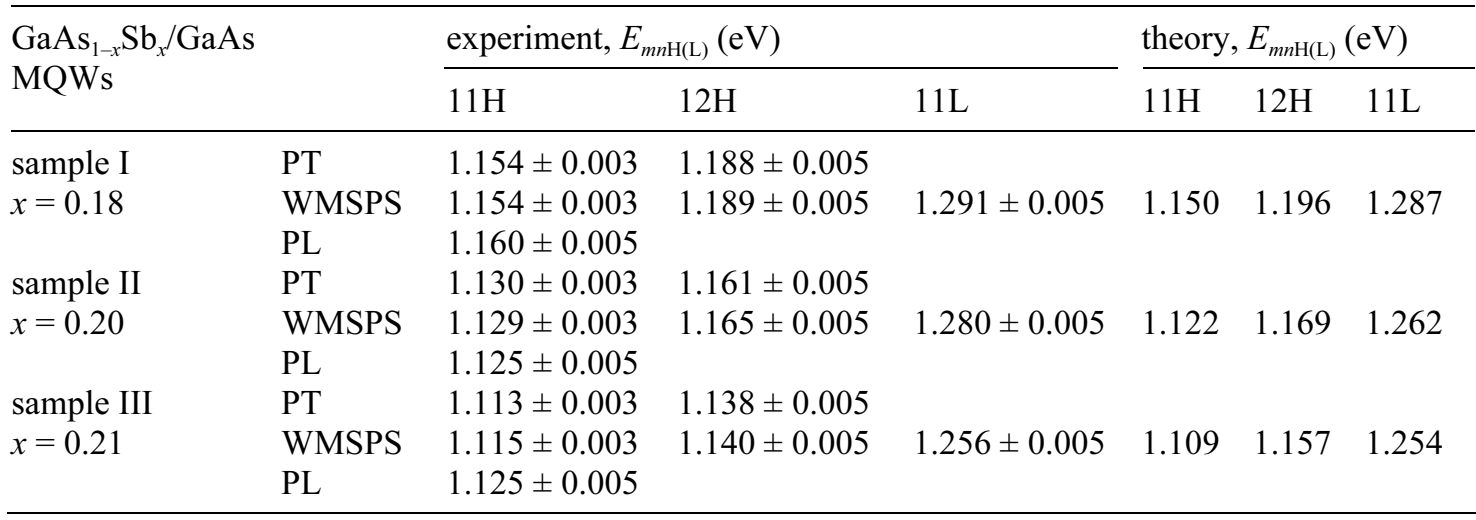

nism involved. PT is a form of electromodulation spectroscopy where the modulation of the electric field in the sample is caused by the photo-excited electron-hole pairs created by the laser diode. In type-II alignment, the positive charges of the holes are confined in the well region and the electrons experience electrostatic attraction toward the well region, it is difficult to achieve electric field modulation effectively in such an indirect transition configuration. Therefore PT becomes less sensitive comparing to WMSPS in the study of type-II structures. The origin of feature $\mathrm{C}$ has been assigned as the first electron state to a light-hole transition and will be discussed in the following section.

Dumitras and Riechert have reported observing a feature located at $78 \mathrm{meV}$ above the PL peak in the derivative SPV spectrum of a type-II GaAs/ $\mathrm{GaAs}_{07} \mathrm{Sb}_{03} / \mathrm{GaAs}$ single quantum well [24]. The origin of this feature was identified as related to transitions between the QW-localized hole state and the extended electron states. By comparing the energy difference of their observed features with that of our observation and the fact that the present samples have lower Sb contents, we can tentatively rule out the assignment for the presently observed feature. In order to specify the observed spectral features, we have calculated the confined energy levels in both the conduction and valence bands by assuming abrupt interfaces. We have solved a one-dimensional Schrödinger equation for five finite square quantum wells based on the envelope function approximation [25] including the effects of strain [26]. A value of unstrained valance band band-offset $Q_{\mathrm{V}}^{0}=0.85$ was used in the calculation [27]. We have used a number of relevant parameters of $\mathrm{GaAs}_{1-x} \mathrm{Sb}_{x}$ listed in [11]. The effective masses of electron, heavy- and light-hole, the lattice constant, hydrostatic (a) and shear (b) deformation potentials, and the elastic stiffness constants $\mathrm{C}_{11}$ and $\mathrm{C}_{12}$ of the ternary material were obtained by linear interpolation of values of the end-point semiconductors GaAs and GaSb [28]. The calculated results are listed in Table 1. A reasonable agreement between experiments and theoretical calculations can be achieved if we identify the features $\mathrm{A}, \mathrm{B}$ and $\mathrm{C}$ to be $11 \mathrm{H}, 12 \mathrm{H}$ and $11 \mathrm{~L}$, respectively. It is noted that the separation between $11 \mathrm{H}$ and $12 \mathrm{H}$ transitions determined from PT/WMSPS reduces as the $\mathrm{Sb}$ exposure time increases. This is different from the theoretical prediction that as the $\mathrm{Sb}$ content increases the separation should become slightly larger. Our observation to the contrary might be due to the intermixing effect at the interfaces and results in a change of the shape of QW profiles and modifies transition energies.

Figure 7 shows CER spectra of the samples in the region of $1.3-1.55 \mathrm{eV}$. The CER spectra exhibit Franz-Keldysh oscillations (FKOs) above the band edge of GaAs. The period of the FKOs is a direct measure of the built-in electric field at the interface of GaAsSb/GaAs [20, 21]. The built-in electric field at the $\mathrm{GaAsSb} / \mathrm{GaAs}$ interfaces increased with the interruption time. An additional feature located below GaAs band edge, denoted as $X$, is observed on the samples under Sb exposure treatment. The probable origin of this feature will be discussed in the following section.

Figure 8 depicts the DC optical bias dependent CER spectra of sample III in the vicinity of GaAs region with an additional steady-state illumination of $670 \mathrm{~nm}$ laser diode as an optical bias. From the 


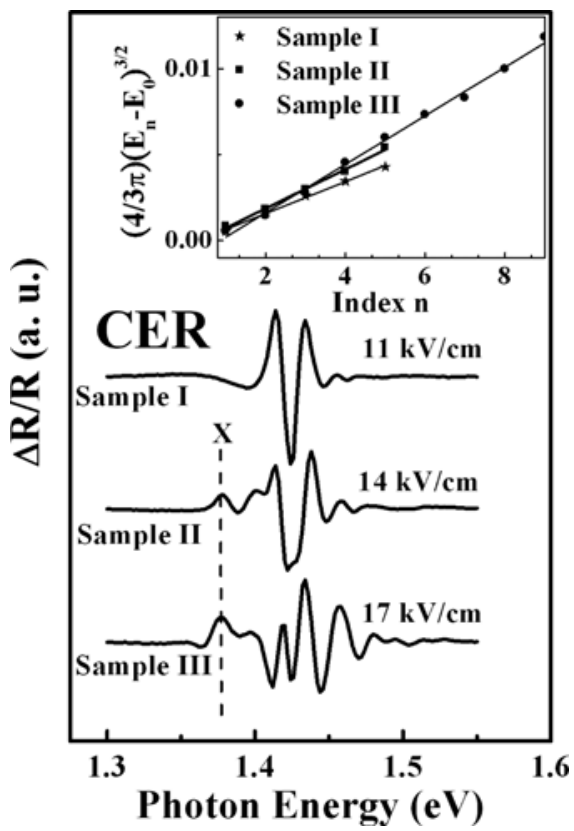

Fig. 7 CER spectra of three samples in the range from $1.3-1.55 \mathrm{eV}$. An additional feature is observed on samples under $\mathrm{Sb}$ exposure treatments. The inset show plots of $(4 / 3 \pi)\left(E_{n}-E_{0}\right)^{3 / 2}$ as a function of the index $n$ which labels the extrema.

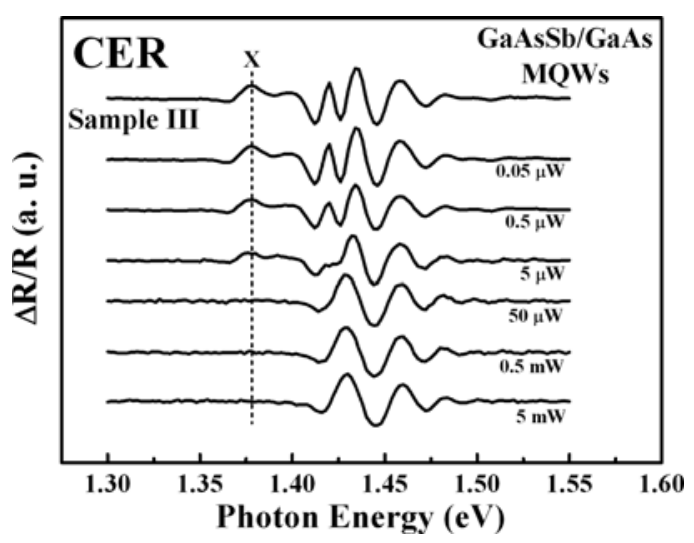

Fig. 8 DC optical bias dependent CER spectra of sample III in the vicinity of GaAs region. The built-in electric field increases slightly and the additional feature disappeared for DC bias exceeding $50 \mu \mathrm{W}$.

CER spectra, FKOs above the GaAs band edge were observed. In contrast to most of the cases, the builtin electric field determined from the period of the FKOs increases slightly with the increase of the intensity of the DC laser bias. The increase of the built-in electric field with the increase of the intensity of the DC laser can be understood as follows: As shown in Fig. 4 the energy minima for electrons and holes lie in different layers. This means that spatially separated electrons and holes are easily realized in such a system, in which electrons are confined in the GaAs layer and holes are localized in the GaAsSb layer. Due to the appearance of the interface states as a consequence of $\mathrm{Sb}$ absorption at the interface and/or the possible interchanging reaction between the $\mathrm{Sb}$ atoms and the As atoms, it is difficult to grow $\mathrm{GaAsSb} / \mathrm{GaAs} \mathrm{MQWs}$ with sharp interfaces. Therefore in the absence of DC optical bias, we always have a slight band bending for the type-II structures. With the DC laser illumination, the photo-generated electrons can move across the interface of $\mathrm{GaAsSb} / \mathrm{GaAs}$, toward the GaAs layer region and leaving behind holes in the GaAsSb region. A direct consequence of this electron-hole separation is an increase in the band bending and the built-in electric field at the interface.

As shown in Fig. 8, without DC laser bias a feature denoted as $X$ located around $1.375 \mathrm{eV}$ is clearly visible. The intensity of this feature gradually decreases with increasing illumination of the optical bias and can be eliminated completely for optical bias exceeds $50 \mu \mathrm{W}$. The same phenomenon is also being observed in sample II. The additional feature only observed on the samples under Sb exposure treatment and its location is lower than that of GaAs impurity levels [29] and thus ruling out the possibility that it belongs to a sub-bandgap feature of GaAs. In this study, we have tentatively assumed the feature to be originated from the transition between the valence band of the GaAs barrier to the localized interface states due to the presence of $\mathrm{Sb}$. Our tentative assertion is based on the observation of the complete elimination of this feature for optical bias exceeding $50 \mu \mathrm{W}$. The steady-state optical illumination of the sample may result in a completely filled interface states as the power exceeds $50 \mu \mathrm{W}$. When the interface 
states are completely occupied, the transition between the valence band of the GaAs barrier and the interface states is prohibited, resulting in the disappearance of the feature in the optical-biased CER spectra. However the origin of this feature warrants further verification and more studies are required.

\section{Summary}

In summary we report a temperature dependent PL and room-temperature modulation spectroscopy study on $\mathrm{GaAsSb} / \mathrm{GaAs} \mathrm{MQWs}$ with different interface treatments. The results indicate that $\mathrm{Sb}$ exposure growth interruptions change significantly the states of the interfaces and thus can influence the optical response. The anomalous temperature dependent PL spectra has been attributed to the carrier localization effect resulting from the presence of Sb clusters and/or Sb content fluctuations at the QW interfaces. Red-shifts of the interband related features and a broader lineshape of the fundamental transition are observed with increasing interface exposure time. The results may be due to an increase of the Sb content and intermixing of $\mathrm{Sb}$ at the GaAs interface layers in the samples. An additional feature has also been observed below the GaAs band energy region in $\mathrm{Sb}$ exposure treated samples and the physical origin of the feature is tentatively attributed to the transition between the valence band of the GaAs barrier and the localized interface states due to the presence of $\mathrm{Sb}$.

Acknowledgements The authors acknowledge the financial support from National Science Council of Taiwan under project No. NSC94-2215-E-011-002.

\section{References}

[1] H. G. Liu, J. Q. Wu, N. Tao, A. V. Girth, E. M. Griswold, T. W. MacElwee, and C. R. Bolognesi, J. Cryst. Growth 267, 592 (2004).

[2] J. E. Cunnningham, M. Dinu, J. Shah, F. Quochi, D. Kilper, W. Y. Jan, M. D. Williams, A. Mills, and W. E. Henderson, J. Vac. Sci. Technol. B 19, 1948 (2001).

[3] P. W. Liu, G. H. Liao, and H. H. Lin, Electron. Lett. 38, 1354 (2002).

[4] F. Quochi, D. C. Kilper, J. E. Cunningham, M. Dinu, and J. Shah, IEEE Photonics Technol. Lett. 13, 921 (2001).

[5] M. Dinu, J. E. Cunningham, F. Quochi, and J. Shah, J. Appl. Phys. 94, 1506 (2003).

[6] X. Sun, S. Wang, J. Hsu, R. Sidhu, X. G. Zheng, X. Li, J. C. Campbell, and A. L. Holmes, J. Sel. Top. Quantum Electron. 8, 817 (2002).

[7] X. Sun, J. Hsu, X. G. Zheng, J. C. Campbell, and A. L. Holmes, IEEE Photonics Technol. Lett. 14, 681 (2002).

[8] M. Yanada, T. Anan, K. Tokutome, A. Kamei, K. Nishi, and S. Sugou, IEEE Photonics Technol. Lett. 12, 774 (2000).

[9] T. Anan, M. Yamada, K. Nishi, K. Kurihara, K. Tikutome, A. Kamei, and S. Sugou, Electron. Lett. 37, 566 (2001).

[10] M. Yano, M. Ashida, A. Kawaguchi, Y. Iwai, and M. Inoue, J. Vac. Sci. Technol. B 7, 199 (1989).

[11] Y. S. Chiu, M. H. Ya, W. S. Su, and Y. F. Chen, J. Appl. Phys. 92, 5810 (2002).

[12] G. Liu, S. L. Chuang, and S. H. Park, J. Appl. Phys. 88, 5554 (2000).

[13] Y. S. Huang and F. H. Pollak, phys. stat. sol. (a) 202, 1193 (2005).

[14] S. Shirakata, M. Kondow, and T. Kitatani, Appl. Phys. Lett. 79, 54 (2001).

[15] T. H. Chen, Y. S. Huang, D. Y. Lin, and K. K. Tiong, J. Appl. Phys. 96, 6298 (2004).

[16] C. K. Kim and Y. H. Lee, Appl. Phys. Lett. 79, 3038 (2001).

[17] M. O. Manasreh, D. J. Friedman, W. Q. Ma, C. L. Workman, C. E. George, and G. J. Salamo, Appl. Phys. Lett. 82, 514 (2003).

[18] R. Kudrawiec, G. Sek, J. Misiewicz, L. H. Li, and J. C. Harman, Appl. Phys. Lett. 83, 1379 (2003).

[19] A. Hoffmann, R. Heitz, A. Kaschner, T. Luttgert, H. Born, A. Y. Qgorov, and H. Riechert, Mater. Sci. Eng. B 93, 55 (2002).

[20] F. H. Pollak and H. Shen, Mater. Sci. Eng. R 10, 275 (1993).

[21] D. E. Aspnes, in: Handbook on Semiconductors, Vol. 2, edited by T. S. Moss (North-Holland, New York, 1980), p. 109.

[22] M. S. Hybertsen, Phys. Rev. Lett. 64, 1984 (1990). 
[23] J. S. Nelson, S. R. Kurtz, L. R. Dawson, and J. A. Lott, Appl. Phys. Lett. 57, 578 (1990).

[24] Gh. Dumitras and H. Riechert, J. Appl. Phys. 94, 3955 (2003).

[25] G. Bastard, Phys. Rev. B 24, 5693 (1981).

[26] S. L. Chuang, Physics of Optoelectronic Devices (Wiley, New York, 1995), p. 144.

[27] T. T. Chen, C. H. Chen, W. Z. Cheng, W. S. Su, M. H. Ya, Y. F. Chen, P. W. Liu, and H. H. Lin, J. Appl. Phys. 93, 9655 (2003)

[28] K. H. Hellwege (ed.), Numerical Data and Functional Relationships in Science and Technology, Landolt Bőrnstein, New Series Group III, Vol. 17a (Springer, Berlin, 1982).

K. H. Hellwege (ed.), Numerical Data and Functional Relationships in Science and Technology, Landolt Börnstein, New Series Group III, Vol. 22a (Springer, Berlin, 1982).

[29] R. L. Tober, J. Pamulapati, J. E. Oh, and P. K. Bhattacharya, J. Appl. Phys. 68, 6388 (1990). 